\title{
Generation of Moisture Reference Years for Interstitial Condensation Risk Assessment: Influence of the Meteorological Record Length
}

\author{
Michele Libralato $^{1}$, Giovanni Murano ${ }^{2}$, Alessandra De Angelis ${ }^{1}$, \\ Onorio Saro ${ }^{1}$, Vincenzo Corrado ${ }^{2}$ \\ ${ }^{1}$ Polytechnic Department of Engineering and Architecture, \\ Università degli Studi di Udine, Italy \\ ${ }^{2}$ Department of Energy, Politecnico di Torino, Italy
}

\begin{abstract}
Heat, air and moisture (HAM) transfer simulations used in moisture accumulation risk assessment of building envelopes require adequate weather files. The common approach is to use weather data of reference years constructed from meteorological records. The length of the records affects the capability of representing the real weather of the resulting reference years. In this paper the problem of the influence of the length of the records on the representativeness of the reference years is addressed and an evaluation of the effect on the moisture accumulation risk analysis with the Glaser Method and with Delphin is presented confirming that records shorter than 10 years could lead to less representative reference years. An alternative reference year (Moisture Reference Year) for moisture related simulations is presented and, the results for the considered weather stations show that it is possible to use it as a representative year for the moisture accumulation risk evaluation.
\end{abstract}

\section{Introduction}

Energy efficiency is a increasingly imposed requirement for new buildings and renovations. One of the possibilities to achieve low energy consumptions is to increase the thermal resistance of the external walls. Adding layers of insulation to existing building envelopes leads to lower thermal dispersion. It is common, when designing the renovation of historical buildings, to add internal insulation, and to be forced to limit the layer thickness due to negative results of moisture accumulation or mould growth risk assessment. The Italian legislation (Italian Republic (2016)) requires the designer to assess the moisture accumulation risk by means of the national edition of standards ISO 13788 (2012) or EN 15026 (2007). The former describes the application of the Glaser method, while the latter, defines the requirements and procedures for the advanced simulation of heat, water vapour and liquid water transfer in building materials described as porous media. In both cases, the simulations require the use of a year of weather data values as boundary conditions. For the advanced heat and moisture migration model, the hourly val- ues of dry-bulb air temperature, water vapour pressure, global solar irradiance, wind speed, wind direction and rainfall of a reference year should be used. The reference year could be designed with the procedure described in ISO 15927-4 (2005), while the Glaser Method uses monthly mean values of the drybulb air temperature and water vapour pressure, in accordance with ISO 15927-1 (2003).

\section{Meteorological record length}

The boundary conditions are, along with the material properties, one of the main sources of inaccuracy of the risk assessments. The weather files could be chosen with the aim of representing the common climate and an average or reference year could be used, or it could be intended to describe extreme events, and, in this case, a critical year should be used. Most of the weather stations have produced relatively long series of measurements of dry-bulb air temperature, relative humidity, solar global irradiance and wind speed, but have only recently begun measuring rainfall and wind direction. The latter values are rarely used in building simulations, but are needed in advanced hygrothermal analysis.

The Typical Meteorological Year (TMY), defined in ISO 15927-4 (2005) procedure, is designed with the application of the Finkelstein-Schafer statistical method on a meteorological record or multi-year (MY). The TMY is the result of the composition of twelve calendar months selected from the MY by the evaluation of their goodness-of-fit to the MY. To achieve a representative TMY, ISO 15927-4 (2005) suggests to use a multi-year of 10 years or longer. This requirement could be very restrictive, for example for recently installed weather stations. The aim of this work is to investigate the influence of the MY length on the moisture accumulation risk assessment performed using reference years for four cities of the Northern Italy. In addition, a reference year, alternative to the TMY for the moisture related risk analysis is presented.

\section{Moisture Reference Year}

The TMY generation procedure is performed considering only the variables relevant to building energy 
simulations (rain is neglected). For this reason, an alternative reference year is proposed, the Moisture Reference Year (MRY), which is designed including the rainfall intensity and duration. For its definition, the resulting MRY has not to be considered as a critical year, but the most representative of the whole MY. Using MRY for moisture related simulations instead of critical years can lead to a valid risk indicator for non-extreme weathers and for the simulation of drying processes in which a critical reference year could lead to overestimations of the risk.

The most recent extended set of reference weather files for the Italian climate has been published by the Italian Thermotechnical Committee (CTI) in 2016, using the method reported in Riva et al. (2010), in accordance with ISO 15927-4 (2005). Further research has been carried out on the topic, extending the TMY generation methodology using on weighting coefficients, for example in Pernigotto et al. (2014), which provided a study on the representativeness of reference years obtained from sequentially reduced MY. A more recent study on the use weighting coefficients is reported in Murano et al. (2018).

In literature several alternatives to the TMY have been proposed for moisture related risk assessments, designed as critical years to obtain the $10 \%$ of failure level. Two alternative approaches could be used: the construction-dependent approach, based on the building features and characteristics, and the construction-independent approach, that does not depend on building characteristics and generates a reference year from the only weather data information. In Kalamees and Vinha (2004) the constructionindependent approach has been used, evaluating the considered weather files with the saturation deficit, an auxiliary parameter calculated from the climate variables, used to identify critical weather files to be used in the mould risk assessment and in the interstitial condensation risk assessment. These reference climate files could be used for the assessment of every building envelope. The constructiondependent weather file selection presented in Zhou et al. (2016), performs first a preliminary selection of three weather reference years based on the Climate Index, a construction-independent auxiliary variable, then the reference year is chosen comparing the results of the simulations of the considered building envelope. The procedure to obtain the risk assessment is more reliable, but the weather file used for the simulation could not be used for other building structures and every different risk assessment would require the computational cost of the weather selection simulations. In order to provide a typical year for moisture transfer simulations, Libralato et al. (2018) extended the ISO 15927-4 (2005) procedure to rainfall, studying the effect of the changing the set of variables used in the MRY generation, comparing the results with moisture transfer simulations on building envelopes. The MRY generation procedure presented in this work considers in addition to the rainfall intensity also an auxiliary variable, the rainfall duration, in the set of MRY primary generating variables.

\section{Theory}

The standardised methodology described by ISO 15927-4 (2005) applies the Finkelstein-Schafer statistic (Finkelstein and Schafer, 1971) on a set of primary parameters $(p)$ which are selected as the most influential in the studied problem. The primary parameters used in ISO 15927-4 (2005) for the generation of the TMY for energy building simulations are the dry-bulb air temperature $(T)$, the global solar irradiance $(I)$, the water vapour pressure $(V P)$ (also the relative humidity, the air absolute humidity and the dew point temperature could be used). This set of primary variables is used to rank the MY months by representativeness using the Finkelstein-Schafer statistic. In addition, a secondary parameter is considered, the wind speed, to perform a secondary selection on the representativeness ranking and select the TMY month.

To build the MRY presented in this work the set of primary parameters is extended including also rainfall intensity $(R I)$ and rainfall duration $(R D)$, two of the most influential variables in heat and moisture transfer simulations. Moreover, the secondary selection is not performed. The rainfall duration has been calculated as the number of consecutive hours with rainfall. This choice has been made to consider the fact that low intensity rainfalls with along duration could be more influential on the moisture content of a wall than short high intensity rainfalls.

\section{Methodology for the construction of the MRY}

For the generation of the MRY form the MY, the following procedure has been used:

(a) Calculation the daily means $\bar{p}$ of the primary variables $p$ for the whole MY.

(b) Calculation of the cumulative distribution function $\Phi(p, m(i), i)$ of the daily means $\bar{p}$ over the whole MY for each day $i$ of a selected calendar month $m$, for each $p$. The variable $i$ represents the ordered number of a day in the MY, from 1 to $N$ (number of days in the MY), and it will be used as a time-stamp. The function $\Phi$ is obtained from the ranking $K(\bar{p}, m, i)$ by numbering the values of the distributions of the considered $p$, separately for each $m$ :

$$
\Phi(p, m(i), i)=\frac{K(\bar{p}, m(i), i)}{N+1}
$$

(c) Calculation of the cumulative distribution function of the daily means within each calendar month $m$ of each year $y, F(p, y(i), m(i), i)$ from the rank order $J(\bar{p}, m(i), i)$, obtained ordering the daily means $\bar{p}$ within the calendar month $m$ 
and the year $y$ :

$$
F(p, m(i), i)=\frac{J(\bar{p}, y(i), m(i), i)}{n+1}
$$

where $n$ is the number of days of the $m$ calendar month considered.

(d) The Finkelstein-Shafer statistic is calculated for each $p$ and each calendar month $m$ in the MY as:

$$
\begin{array}{r}
F_{S}(p, y, m)=\sum_{i=1}^{n} \mid F(p, y(i), m(i), i) \\
-\Phi(p, m(i), i \mid
\end{array}
$$

(e) For each $p$, the ranking $R$ is assigned to each calendar month $m$, obtained from the ordering of the $F_{S}(p, y, m)$ of each $y$ separately for each calendar month $m$ :

$$
R(p, y, m)=\frac{L\left(F_{S}\right)}{n_{y}+1}
$$

with $n_{y}$ the number of years of the MY.

(f) The ranking $R$ of each calendar month is calculated for all the primary parameters and then summed, to obtain the total ranking $R_{t o t}$ :

$$
\begin{array}{r}
R_{\text {tot }}(y, m)=R(T, y, m)+R(V P, y, m) \\
+R(I, y, m)+R(R I, y, m)+R(R D, y, m)
\end{array}
$$

(g) Each calendar month $m$ of the MRY is chosen among the months of the MY as the month $m$ of the year $y$ with the lowest $R_{t o t}$.

(h) The MRY is composed by the hourly series of the weather variables of the selected months and the continuity between every month is set with a linear interpolation, in order to provide a smooth transition between months from different years.

\section{Comment on the Rainfall Duration}

As seen at the step (a) of the MRY design procedure, the weather variables are not considered as hourly values, but as daily means, loosing some information about the distribution in time of the variable. With this calculation, a rain event of two consecutive hours is not distinguishable from two separate rain events of the same overall intensity, while the difference between the two effects on the analysed wall is different. For this reason, the auxiliary variable $R D$, rainfall duration, has been introduced in the set of primary parameters.

\section{Calculation}

The MYs measured by four weather stations of Northern Italy have been considered, the cities and the weather stations locations are listed in Table 1. The MRY and TMY have been designed for every station, considering, when possible, 5 different MY lengths:
1996-2017, 1996-2007, 2002-2017, 2007-2017, 20122017. The obtained weather files are compared and then evaluated by performing moisture accumulation risk analysis on six walls.

\section{Weather data set}

The periods of missing and invalid values have been replaced with different interpolation techniques, depending on the duration of the missing data. The missing data of the wind speed for the period from the 1996 to 2011 for the station of Bergamo have been substituted with the scaled wind speed measurements of another weather station of Bergamo. To normalize the wind speed values of the measurements of the second weather station, they have been scaled of a factor equal to the rate between the means of the wind speed in the two locations. Due to an excessive lack of data, the wind direction measurements for the city of Bergamo have not been considered.

Table 1: Positions of the considered weather station and length of the Multi-year record.

\begin{tabular}{ccccc}
\hline Station & $\begin{array}{c}\text { Lat. } \\
\left({ }^{\circ}\right)\end{array}$ & $\begin{array}{c}\text { Long. } \\
\left({ }^{\circ}\right)\end{array}$ & $\begin{array}{c}\text { Alt. } \\
(\mathrm{m} \text { a.s.l. })\end{array}$ & MY years \\
\hline Aosta & 45.75 & 7.68 & 569 & $1996-2017$ \\
\hline Bergamo & 45.66 & 9.66 & 211 & $1996-2017$ \\
\hline Torino & 44.96 & 7.71 & 226 & $2002-2017$ \\
\hline Udine & 46.03 & 13.23 & 91 & $1996-2017$ \\
\hline
\end{tabular}

In the following plots the qualitative evolutions of the rainfall intensity and rainfall duration are presented and compared with that of the temperature. Figure 1 shows the rainfall intensity annual means of the MY of the four stations and the rainfall duration annual mean, calculated as the sum of the annual hours of rain divided by the number of hours of the year. The two plots, even if the two variables are different, show a similar behaviour, with some exceptions. These are due to high intensity rainfalls with a duration of less than an hour, that could be less influential on the moisture content of building materials than long-lasting low intensity rains. In order to give more relevance in the reference year construction proces the rainfall duration has been included as a primary parameter.

In Figure 2 the annual mean rainfall intensity in Udine is presented. The values are calculated as the total rainfall of the year divided by the number of hours of the year. The relatively large standard deviation shows that the $R I$ has a large variability compared to the one of the other variables.

For example, the air dry-bulb temperature plot in Figure 3 shows a relatively small standard deviation, indicating less extreme values and a more regular trend. Similar behaviours have been observed for the other stations.

This behaviour is explained in Figure 4 and in Figure 5 , where the single year are plotted separately in order to appreciate the irregular distribution of the 

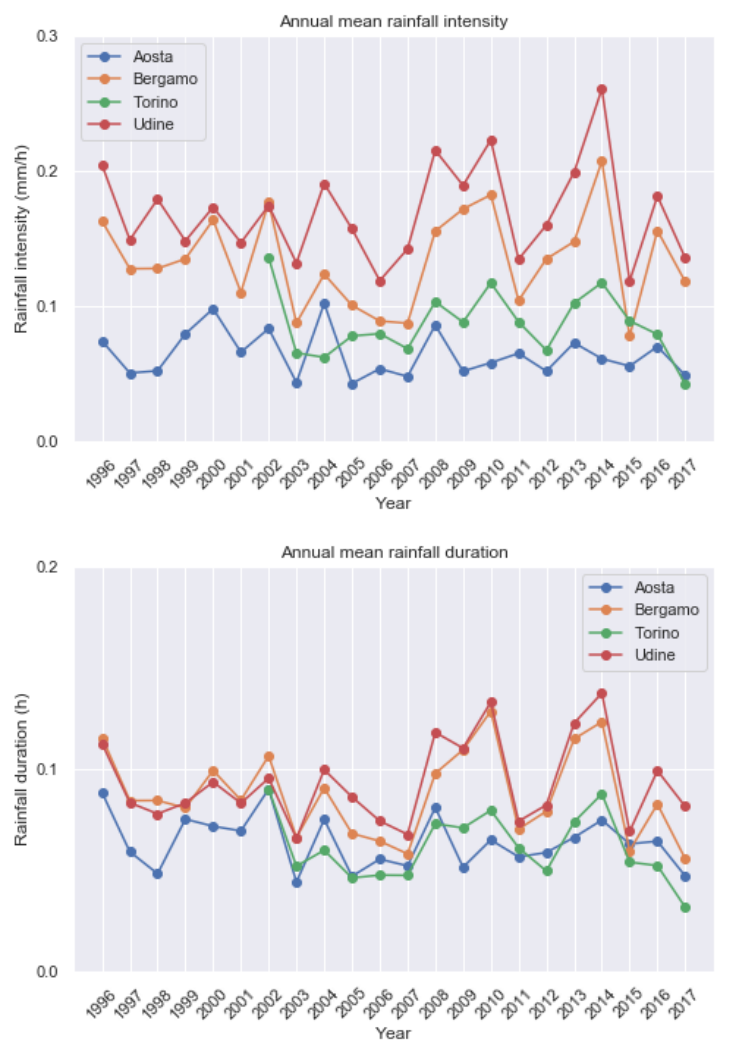

Figure 1: Annual mean rainfall intensity and annual mean rainfall duration for the four stations considered.

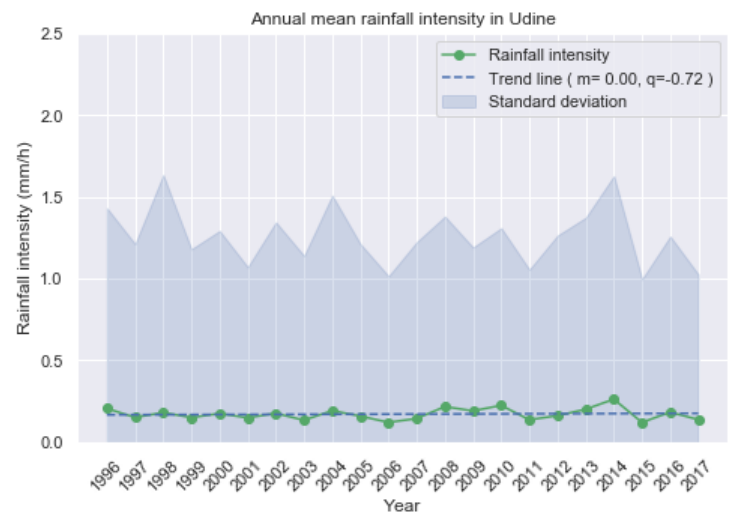

Figure 2: Annual mean rainfall intensity in Udine, linear trend and standard deviation calculated on the hourly values.

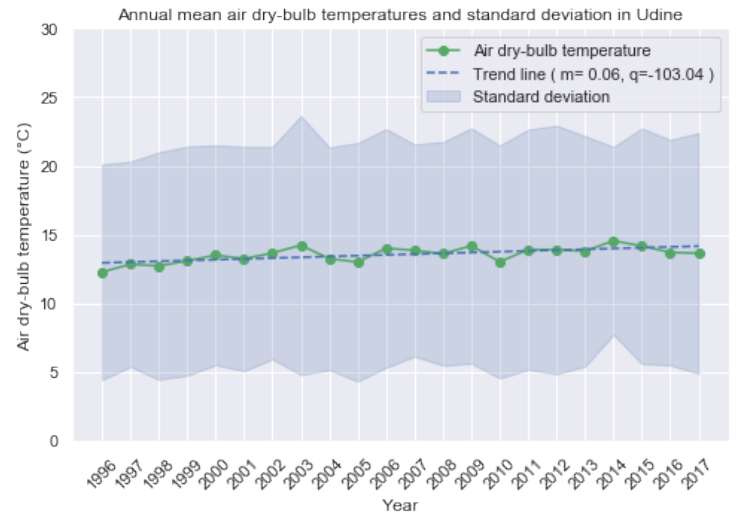

Figure 3: Annual mean dry-bulb air temperature in Udine, linear trend and standard deviation calculated on the hourly values.

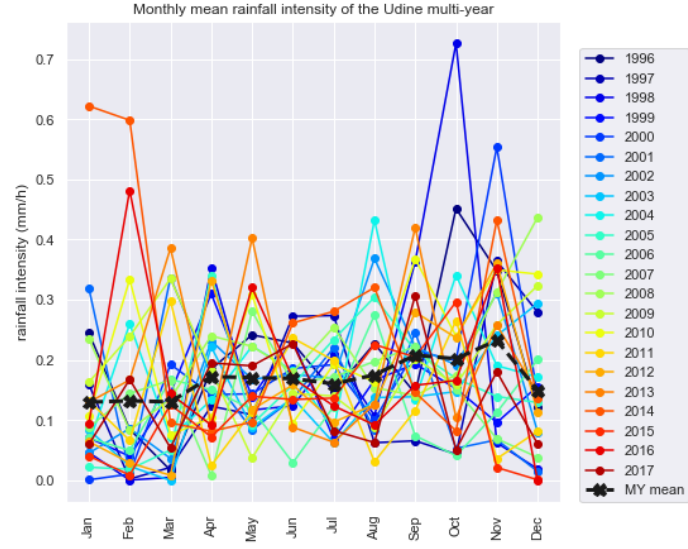

Figure 4: Monthly mean rainfall intensity in Udine for each year considered in the MY compared to the mean of every calendar month of the multi-year (MY mean).

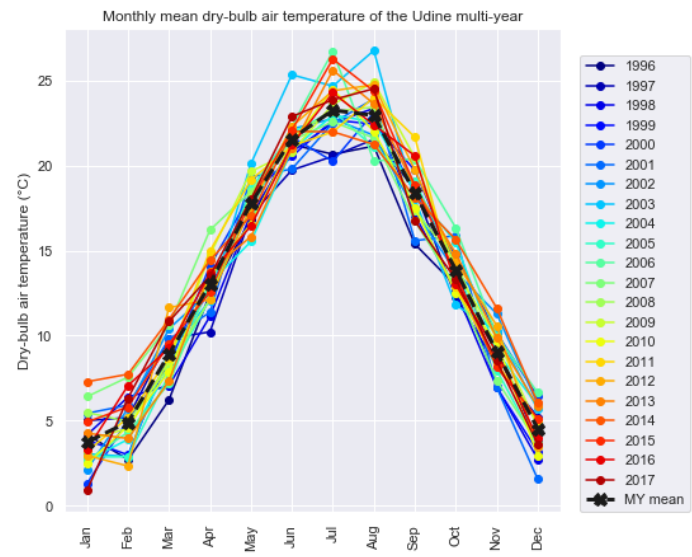

Figure 5: Monthly mean air dry bulb temperature in Udine for each year considered in the MY compared to the mean of every calendar month of the multi-year (MY mean).

peaks among the months. They show the monthly mean values of the whole MY respectively of air dry bulb temperature and rainfall intensity. The air bulb temperature curves of every year match closely the MY mean, denoting an analogous behaviour, while the rainfall intensity has relatively larger variations from the MY mean.

\section{Case studies}

The reference years produced in this study have been evaluated for the use in moisture accumulation risk assessment. The evaluation of the moisture content has been performed using a simplified method, the Glaser Method, and an advanced model, using the software Delphin 6 (Sontag et al., 2013). The first method considers the air dry-bulb temperature and the relative humidity, while the advanced model considers also the rainfall intensity, solar global irradiance, and, if required, the wind speed and direction for the calculation of the driving rain. For the sake of simplicity, in this evaluation, the wind speed and direction have not been used, and the whole rainfall 
intensity has been considered as driving rain on the wall. With this hypothesis the representativeness of the reference years could be evaluated with higher moisture contents variations. The evaluation of the risk parameter has been performed with a simplified method for both simulation procedures. The risk $P_{y}^{G}$ for the Glaser method $(G)$ over the period $y$ has been calculated evaluating the ratio between $n_{m \mid \varphi=1}$, number of months with liquid water between the layers (with the relative humidity equal to 1 ), and the total number of months $N_{m}$ of $y$ as in Eq. 6 .

$$
P_{y}^{G}=\frac{n_{m \mid \varphi=1}}{N_{m}}
$$

The evaluation for the MY has been performed considering all the monthly mean variables for all the months of the MY, while, the assessment for the reference years has been performed for a period of five years. The assessment of the risk $P_{y}^{D}$ for the advanced simulation method has been performed using the same number of years. Five years is the time required in the Delphin simulations for the total moisture content of the wall at the first time-step of the year to be equivalent to the moisture content of the first time-step of the following year. The risk $P_{y}^{D}$ has been calculated as the ratio between $n_{h \mid \varphi=0.95}$, number of hours with values of relative humidity higher than 0.95 on an internal layer of the wall, and $N_{h}$, the total number of hours considered in the simulation (Eq. 7).

$$
P_{y}^{D}=\frac{n_{h \mid \varphi=0.95}}{N_{h}}
$$

The external layer has not been considered for this evaluation. The indoor and outdoor surface boundary conditions for the Delphin simulations are set in accordance with EN 15026 (2007), the internal environment is set to the "normal occupancy" conditions described in the standard. The Glaser method boundary conditions are set in accordance with the ISO 13788 (2012), for a continental climate with "normal occupancy". The material properties are taken from the Delphin material database. In both approaches the internal surface thermal resistance is set to $0.25 \mathrm{~m}^{2} \cdot \mathrm{K} / \mathrm{W}$ and the external thermal resistance to $0.04 \mathrm{~m}^{2} \cdot \mathrm{K} / \mathrm{W}$. In the Delphin simulations the surface vapour exchange equivalent air layer thickness is set to $0.003 \mathrm{~m}$ on the outside and to $0.008 \mathrm{~m}$ on the inside. For both simulation procedures, a risk of 0 indicates that the water condensation has never occurred, while a risk equal to 1 , denotes the presence of liquid water in a layer of the wall in every time-step of the simulation period. The risk assessment has been performed for six walls, typical of the Northern Italy regions (Ballarini et al., 2014), redesigned to have liquid water condensation and accumulation between the material layers. The walls are described in Figure 6. The wall properties are summarized in Table 2 .
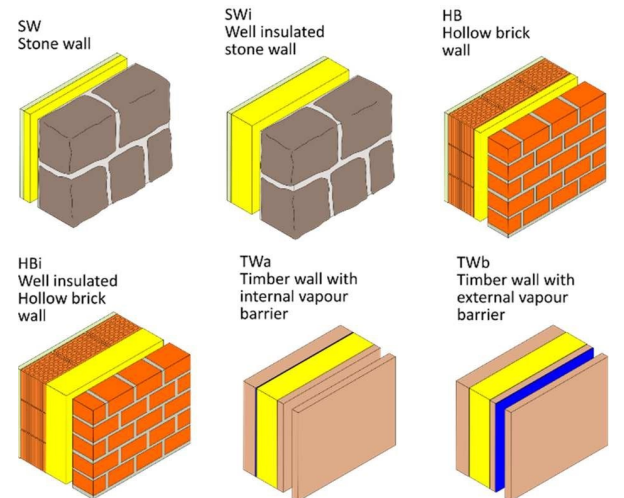

TWb

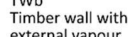

extern
barrier
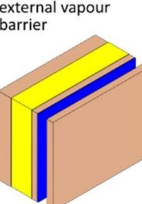

Figure 6: Building envelopes considered in the study. The exterior surface is on the right.

Table 2: Wall thickness d, Thermal transmittance $U$ and equivalent air layer thickness $S_{d}$ of the walls considered for the risk assessment evaluations. The walls are identified in Figure 6.

\begin{tabular}{lccr}
\hline \multicolumn{1}{c}{ Id. } & $\begin{array}{c}d \\
(\mathrm{~m})\end{array}$ & $\begin{array}{c}U \\
\left(\mathrm{~W} / \mathrm{m}^{2} \mathrm{~K}\right)\end{array}$ & $\begin{array}{c}S_{d} \\
(\mathrm{~m})\end{array}$ \\
\hline SW & 0.38 & 0.70 & 5 \\
SWi & 0.53 & 0.13 & 50 \\
$\mathrm{HB}$ & 0.49 & 0.39 & 7 \\
$\mathrm{HBi}$ & 0.58 & 0.15 & 41 \\
TWa & 0.53 & 0.13 & 56 \\
TWb & 0.53 & 0.13 & 56 \\
\hline
\end{tabular}

\section{Results and discussion}

The MRY and TMY generated from the five different MY tend to have rainfall intensities closer to the MY mean values. The months with the extreme values are excluded by the ranking procedure in both reference year generation methods. The same behaviour is found considering the rainfall intensity annual mean values in Figure 8 and for the rainfall duration. In Figure 9 the rainfall intensity monthly mean value is presented for the five MY considered, showing that a common evolution is not found. This behaviour of the variable could prevent the statistical framework used in this work from identifying a main trend to be represented by the reference years. The results of the evaluation are presented in Figures 10 and 11 . The values presented in the plots are differences $\Delta P^{I}$ (defined in Eq. 8) between the risk $P_{R Y}^{I}$ calculated with the use of a reference year (specified on the $\mathrm{x}$ axis) and the risk $P_{M Y}^{I}$, obtained using the MY from the year as boundary conditions. In order to be representative, the reference years, should provide interstitial condensation risk values close to the ones obtained with the MY. Larger $\Delta P^{I}$ values indicate less representative reference years. The superscript $I$ indicates the calculation method used: $G$ for the Glaser method and $D$ for the Delphin simulation.

$$
\Delta P^{I}=P_{R Y}^{I}-P_{M Y}^{I}
$$

The MY for the stations of Aosta, Bergamo and 
Udine is from the year 1996 to 2017 , while the one for Torino is form the 2002 to 2017. Positive values of $\Delta P^{I}$ indicate higher $P_{R Y}^{I}$ values, meaning that the reference year simulation is conservative. The Delphin simulations performed using the reference years are generally in accordance with the MY simulations. The exceptions are visible in the plot for the station of Aosta in Figure 10, where the MRY obtained with the records 1996-2017, 2002-2018, 2007-2017 of the station of Aosta produce over-conservative results for the $\mathrm{HB}, \mathrm{HBi}, \mathrm{SW}$ walls (the wall identifiers are listed in Figure 6). The reference years obtained from the station of Bergamo are generally in accordance with the MY simulations except for the not acceptable underestimations given by the MRY from the records 1996-2007, 2002-2007 and 2012-2017 and the TMY from the 2002-2017 and 2012-2017. For the station of Torino the results of the reference years are generally in accordance with the MY risks with the exception of the MRY 2012-2017 that underestimates the risk. The MRY 2002-2017 and 2007-2017 and the TMY 2007-2017 are overestimating the risk in a relevant manner for the SWi wall. The results of the reference years for the station of Udine are generally conservative respect to the MY. The most representative results are given by the MRY 1996-2017 and by the TMY 1996-2007. The Glaser method results, presented in Figure 11, denote a general good agreement between the reference years and the MY, for every station. The results for the station of Bergamo are slightly underestimating the risk of moisture accumulation for each reference year, with peaks at the reference years of the period 2012-2017.

\section{Conclusions}

The influence of the meteorological record length for the generation of weather files of reference years has been evaluated for the moisture accumulation risk assessment for four weather stations in Northern Italy: Aosta, Bergamo, Torino and Udine. Five sets of years have been considered as MY for the reference years generation: 1996-2017, 1996-2007, 2002-2017, 2007-2017, 2012-2017. Two reference years construction method have been considered, the TMY used for building energy simulations, defined in ISO 15927-4 (2005), and the MRY, proposed in this work, to be used in heat and moisture transport simulations. It has been found that the TMY and the MRY generation procedures generate years representative of the considered MY. Among the weather variables, it has been observed that the generated rainfall intensity series are representative of the mean behaviour, thanks to the exclusion of the critical months from the resulting years. Afterwards, ten reference years produced for every station have been used for the moisture accumulation risk assessment of six walls with two methods: the Glaser method and with the software Delphin 6. The risks calculated using the MRY

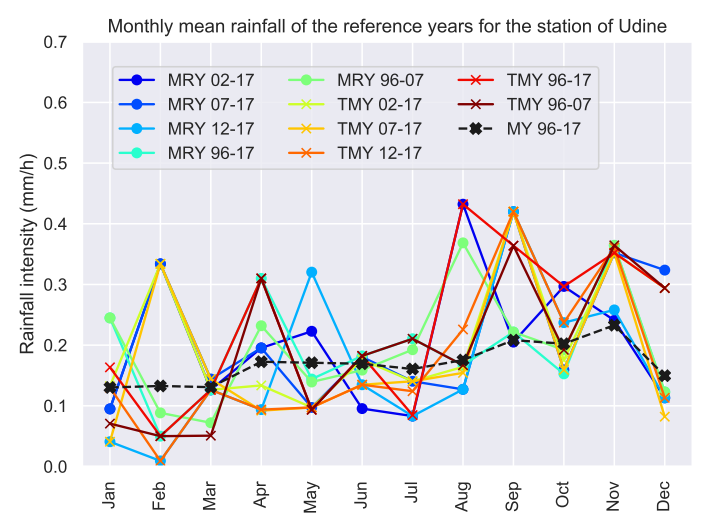

Figure 7: Monthly mean rainfall intensity in Udine for each MRY compared to the overall mean rain intensity of the $M Y$ and its standard deviation.

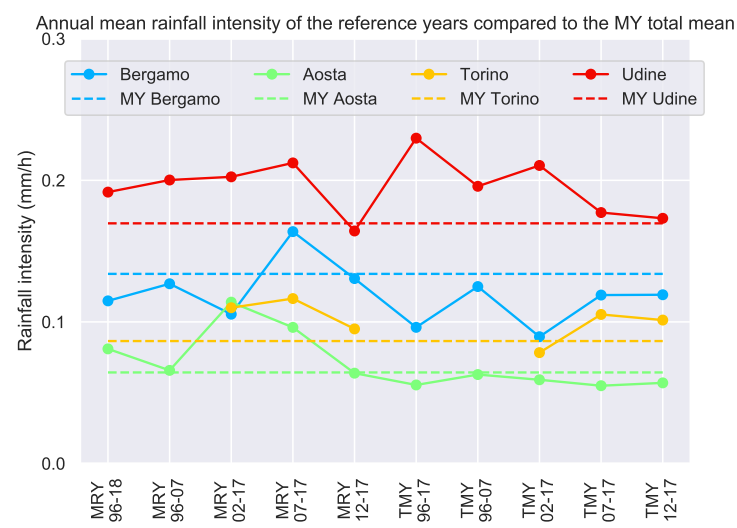

Figure 8: Annual mean rainfall intensity for each $M R Y$ presented compared to the overall mean rain intensity of the multi-year of the four stations considered.

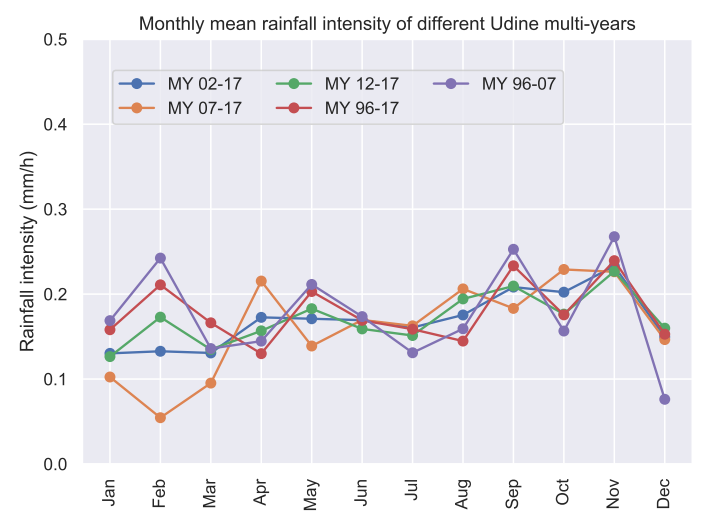

Figure 9: Monthly mean rainfall intensity for the five multi-year considered for the station of Udine. 

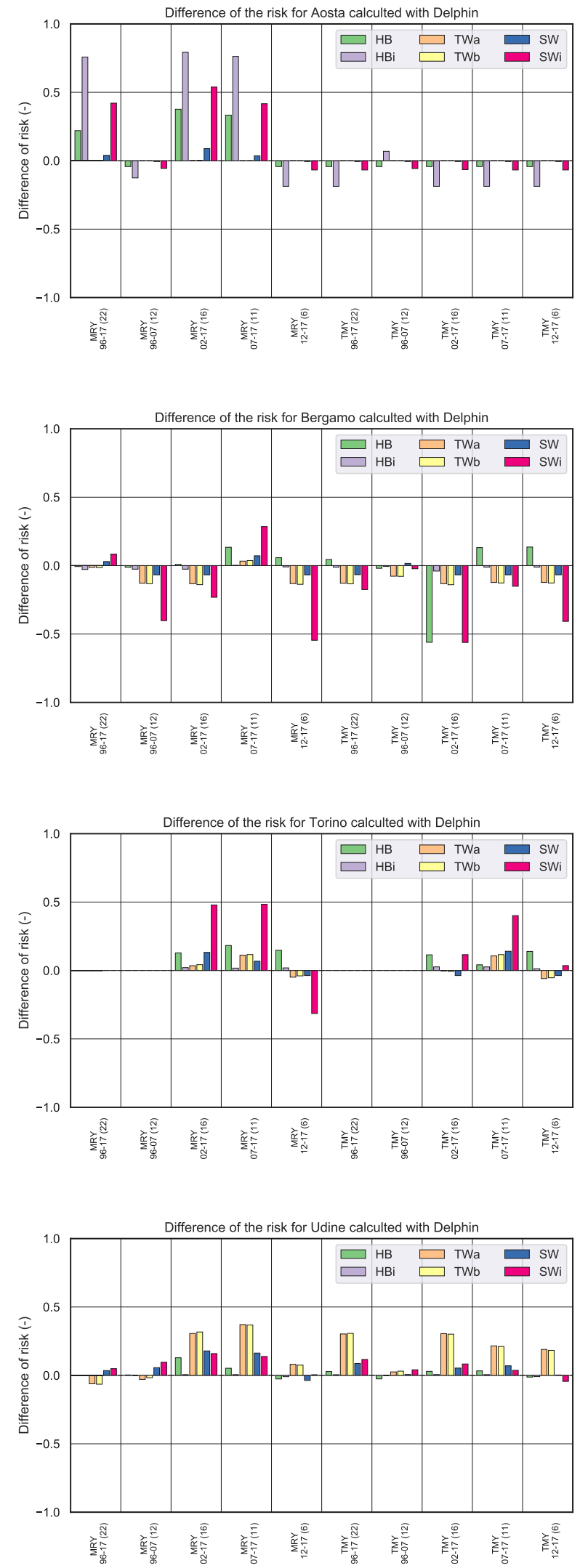

Figure 10: Evaluation of the reference years in terms of moisture accumulation risk calculated with Delphin for the four considered stations.
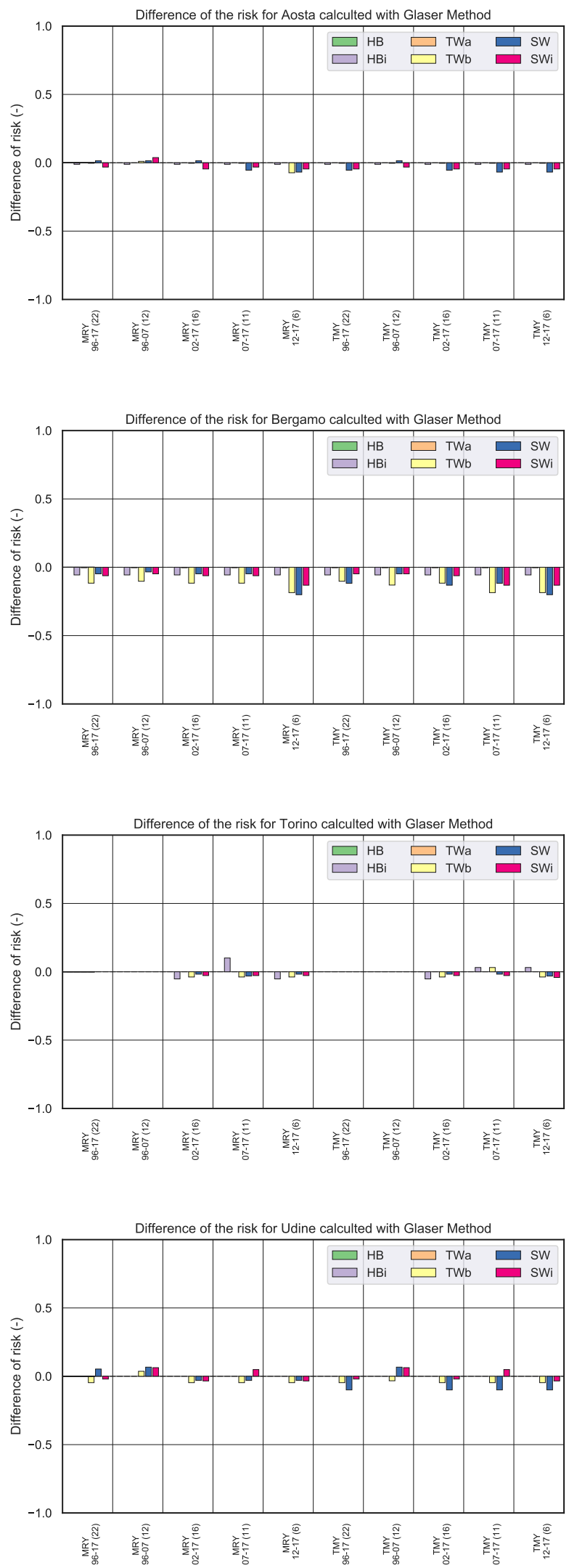

Figure 11: Evaluation of the reference years in terms of moisture accumulation risk calculated with the Glaser method for the considered stations. 
and TMY have been compared with the risk obtained from the full MY. It has been observed that, as a general trend, the MRY and TMY obtained from the full MY produced the most representative results, while the MRY and TMY from the shorter MY intervals resulted to be less representative. In some cases, the reference years obtained from periods shorter than 10 years could be less representative and lead to underestimations of the risks. When the risk is assessed using the Glaser method the differences of risk are less than 0.25 , while for the walls with thicker layers of insulation, the risk differences calculated with Delphin are from 0.5 to 0.75 . As seen in Libralato et al. (2019), when the moisture content is evaluated with HAM models and the rain is considered as a moisture source in the calculation, larger variations of moisture contents could be expected, with larger differences between the resulting risks evaluated. Finally, from these results it could be concluded that the proposed MRY construction method leads to valid reference years that could be used for moisture related calculations in addition to critical years, as representations of the long term mean weather data, for moisture accumulation risk analysis or simulations of drying of building structures. Future work will concentrate on further evaluations of the presented MRY generation procedure for different climate zones and on the development of a design method for critical years for heat and moisture transfer simulations.

\section{Acknowledgements}

The authors thank ARPA Piemonte, ARPA FVG (OSMER), Centro Funzionale della Valle d'Aosta and ARPA Lombardia for supplying the raw weather data and Bauklimatik Dresden Software GmbH for providing the software Delphin 6. This research has been made possible thanks to the $\mathrm{PhD}$ funding of the Provincia di Udine.

\section{References}

Ballarini, I., S. P. Corgnati, and V. Corrado (2014). Use of reference buildings to assess the energy saving potentials of the residential building stock: The experience of tabula project. Energy Policy 68, 273-284.

European Committee for Standardisation (2007). Hygrothermal performance of building components and building elements - Assessment of moisture transfer by numerical simulation (EN 15026:2007).

Finkelstein, J. M. and R. E. Schafer (1971). Improved goodness-of-fit tests. Biometrika 58(3), 641.

International Organisation for Standardisation (2012). Hygrothermal performance of building components and building elements - Internal surface temperature to avoid critical surface humidity and interstitial condensation - Calculation methods (ISO 13788:2012).
International Organisation for Standardisation (2003). Hygrothermal performance of buildings Calculation and presentation of climatic data Part 1: Monthly means of single meteorological elements (ISO 15927-1:2003).

International Organisation for Standardisation (2005). Hygrothermal performance of buildings Calculation and presentation of climatic data- Part 4: Hourly data for assessing the annual energy use for heating and cooling (ISO 15927-4:2005).

Italian Republic (2016). Applicazione delle metodologie di calcolo delle prestazioni energetiche e definizione delle prescrizioni e dei requisiti minimi degli edifici, Ministerial Decree 26 June (in Italian).

Kalamees, T. and J. Vinha (2004). Estonian Climate Analysis for Selecting Moisture Reference Years for Hygrothermal Calculations. Journal of Building Physics.

Libralato, M., G. Murano, O. Saro, and A. D. Angelis (2018). Hygrothermal modelling of building enclosures: reference year design for moisture accumulation and condensation risk assessment. In 7th International Building Physics Conference, IBPC2018.

Libralato, M., O. Saro, A. D. Angelis, and S. Spinazzè (2019). Comparison between Glaser Method and Heat, Air and Moisture Transient Model for Moisture Migration in Building Envelopes. Applied Mechanics and Materials 887, 385-392.

Murano, G., D. Dirutigliano, and V. Corrado (2018, aug). Improved procedure for the construction of a Typical Meteorological Year for assessing the energy need of a residential building. Journal of Building Performance Simulation, 1-14.

Pernigotto, G., A. Prada, A. Gasparella, and J. L. Hensen (2014). Analysis and improvement of the representativeness of en iso 15927-4 reference years for building energy simulation. Journal of Building Performance Simulation 7(6), 391-410.

Riva, G., G. Murano, V. Corrado, P. Baggio, and G. Antonacci (2010). Definizione degli anni tipo climatici delle province di alcune regioni italiane (in Italian). ENEA, Ministero dello Sviluppo Economico, 347 .

Sontag, L., A. Nicolai, and S. Vogelsang (2013, nov). Validierung der Solverimplementierung des hygrothermischen Simulationsprogramms Delphin.

Zhou, X., D. Derome, and J. Carmeliet (2016). Robust moisture reference year methodology for hygrothermal simulations. Building and Environment 110, $23-35$. 